\title{
A NEW APPROACH FOR ACCURACY IMPROVEMENT OF PULSED LIDAR REMOTE SENSING DATA
}

\author{
Guoqing Zhou ${ }^{1}$, Wei Huang ${ }^{1,2}$, Xiang Zhou ${ }^{1,2, *}$, Chaoshuang He ${ }^{1}$, Xiaozhu Li ${ }^{1}$, Yu Huang ${ }^{1}$, Lieping Zhang ${ }^{1,2}$ \\ ${ }^{1}$ Guangxi Key Laboratory of Spatial Information and Geomatics, Guilin University of Technology, No. 12 Jian'gan Road, Guilin, \\ Guangxi 541004, China - gzhou@glut.edu.cn \\ ${ }^{2}$ Department of Mechanical and Control Engineering, Guilin University of Technology, No. 12 Jian'gan Road, Guilin, Guangxi \\ 541004, China - (102016477, zqx0711)@glut.edu.cn
}

Commission III, ICWG III/IVb

KEY WORDS: Time interval measurement, Pulsed lidar, Data quality, Capacitor charge, Voltage curve, Remote sensing

\begin{abstract}
:
In remote sensing applications, the accuracy of time interval measurement is one of the most important parameters that affect the quality of pulsed lidar data. The traditional time interval measurement technique has the disadvantages of low measurement accuracy, complicated circuit structure and large error. A high-precision time interval data cannot be obtained in these traditional methods. In order to obtain higher quality of remote sensing cloud images based on the time interval measurement, a higher accuracy time interval measurement method is proposed. The method is based on charging the capacitance and sampling the change of capacitor voltage at the same time. Firstly, the approximate model of the capacitance voltage curve in the time of flight of pulse is fitted based on the sampled data. Then, the whole charging time is obtained with the fitting function. In this method, only a high-speed A/D sampler and capacitor are required in a single receiving channel, and the collected data is processed directly in the main control unit. The experimental results show that the proposed method can get error less than 3 ps. Compared with other methods, the proposed method improves the time interval accuracy by at least $20 \%$.
\end{abstract}

\section{INTRODUCTION}

Pulsed lidar measurement is a new means of remote sensing, mainly used for laser ranging and point cloud imaging (Zhou, 2009; 2015). Lidar has the advantages of light weight, small size, high reliability, low energy consumption and is widely used in industrial production and environmental monitoring (Zhou, 2014). With the development of remote sensing technology, the accuracy of lidar is getting higher and higher. In order to obtain more accurate and clearer point cloud images, a higher quality lidar data is necessary. There are many factors that affect the accuracy of lidar data, the accuracy of time interval mainly determines the quality of the final point cloud. So, the improvement of lidar data quality can be achieved by improving the accuracy of time interval in the remote sensing. The traditional methods of time interval measurement mainly include analog conversion, digital conversion and insertion. The analog conversion (Caulfield, 1986) cleverly transforms time information into capacitor charging-discharging process, but the errors are difficult to eliminate due to the nonlinear relationship between the voltage and current in the capacitor. The operation principle of the digital conversion method is simple (Gao, 1991), but the time delay occurs when the start-stop timing. The insertion method (Nutt, 1968) is an improvement for the analog conversion and the digital conversion. At present, there is a common insertion delay line interpolation (Kalisz, 2002) and analog interpolation (Zieliski, 2003). The most representative of the delay line insertion method is the time interval measurement chip of TDC series (TDCs-Time-to-Digital Converters, 2017) produced by the German ACAM Inc. The error of this method is mainly derived from the minimum unit of the retarder and the nonlinear relationship between the retarder and the trigger. The simulation insertion method is based on the analog conversion method. The stop signal also makes the capacitor charge and discharge. The accuracy of the analog insertion method is mainly caused by the nonlinear discharge of capacitors. In addition, because of the use of extremely high frequency clocks, it is easy to be jitter and cause errors. Many researchers have made great efforts for high precision time interval measurement. Palojarvi (1999) designed a time interval measurement method that converts time information into capacitive charge and discharge (constant current) processes and then converts the analog signal to a digital signal and calculates the time interval by the formula, a \pm 3.5 ps measurement Accuracy is achieved. Nissinen (2009) designed a circuit that measures the time interval and the slew rate of stop pulse, and compensate for the error produced in the discriminator. The error after compensation is reduced to \pm 30 ps. Klepacki (2014) achieved the single-shot precision of 7.5 ps by combining direct counting with two-stage interpolation in a single clock.

In this paper, a method of charging the capacitance and sampling the fitting voltage curve is proposed to improve the accuracy of time interval measurement in lidar data processing. According to the fitting model of the variation curve of capacitance voltage, the actual time of the capacitor charging is calculated. Experiment shows that the method can minimize the error caused by the delay of start-stop timing and the nonlinear change of capacitance voltage in the measurement process, and improve the quality of lidar data.

\footnotetext{
* Corresponding author: Xiang Zhou; E-mail: zqx0711@glut.edu.cn
} 


\section{PRINCIPLE OF TIME INTERVAL MEASUREMENT}

\subsection{Basic Theory}

In remote sensing applications, pulsed lidar technology realizes the distance measurement by transmitting specific pulses to target and receiving echo from target. Then, the shape of the three-dimensional point cloud of the object can be formed by combining the distance of the feature points on the surface of the object. This point cloud imaging is the latest and widely used remote sensing technology (Zhou, 2004). In time interval measurement, the distance of the target is calculated by measuring the time interval between the laser emission pulse and the echo pulse. The traditional methods of time interval measurement mainly include analog conversion, digital conversion and insertion. These methods are mainly divided into three detailed technical principles. First, the information of the laser pulse time of flight is converted to the form of capacitance charge and discharge. Second, the time of the laser pulse time is directly counted by the clock counter. Third, the above two technologies are combined to reduce the error. It is difficult to eliminate the error due to the existence of the nonlinear properties of the physical devices. Although ACAM Inc. has produced a compact TDC integrated microchips to achieve time interval measurement, but the latest TDC-GP22 series still has a minimum error of $22 \mathrm{ps}$.

\subsection{Time interval Measurement Based on Fitting Algorithm}

In order to minimize the error of the time interval to most, the method of capacitor charging is still used to replace the time information of the TOF. Here only the use of capacitor charging time to analyse the problem, which is different from the traditional charging and discharging. The principle of capacitorbased charging and sample-fitting of the capacitor voltage curve is as follows: First, a capacitor charging and discharging circuit is set after the time discriminating unit, and the value of the capacitor is constant. When starting the measurement, there is a portion of the start pulse that triggers the charging of the capacitor. Then, a microcontroller unit (MCU) controls the high-speed A/D sampler to sample the voltage of the capacitor, and the sampled data is collected into the MCU for processing. Since the TOF varies, so the value of the capacitance should be enough to meet the different measurement distances. Second, when the received signal arrives, the signal generated by the timing discrimination unit stops the charging operation of the capacitor, and at the same time, it notifies the MCU to stop the sampling operation and discharge the capacitor through the circuit control quickly and completely. In this process, the response time from the output of the timing signal to the charging of the capacitor is negligible. However, the response time (including a very short delay) that the signal notifies the controller is generally several tens of nanoseconds (the controller clock frequency is $10 \sim 50 \mathrm{MHz}$ ). A buffer is maintained from charging stop to discharging through the related circuit design, and the capacitor voltage is kept constant in this range, so the A/D sampler can completely sample the voltage value of this gap (voltage at the end of TOF). This operation method can eliminate the trouble of peak-keeping circuit design. Finally, the sampled data is further processed through MCU. According to a series of points on the voltage curve of the sampled capacitor, the suitable fitting model is selected to match these points to get the best voltage change curve function. According to the curve function, the charging times from the zero voltage point to the maximum voltage point are calculated, and this time is the real time interval with minimum error. The calculation of the fitting function can ignore the nonlinear characteristics of the actual voltage variation curve. The capacitance is also affected by the noise and temperature, and there will be a nonlinear change, even if constant current is charged in practical operation. Therefore, general charging measures can be adopted directly.

As shown in Figure 1, the high speed A/D sampler begins to sample the capacitor voltage at a high speed after a period of time lag behind the starting point of the capacitor charging during the charging process. The time axis of the first sampling point can be customized to set up a starting time (suppose $t_{0}$ ). When the sample is finished, these data can be expressed as

$$
F=\left[\begin{array}{ccccc}
P_{1} & P_{2} & P_{3} & \cdots & P_{n} \\
v_{1} & v_{2} & v_{3} & \cdots & v_{n} \\
t_{0} & t_{0}+T & t_{0}+2 T & \cdots & t_{0}+(n-1) T
\end{array}\right]
$$

where $\quad P=$ sampling point

$v=$ voltage value of sampling

$T=$ sampling period

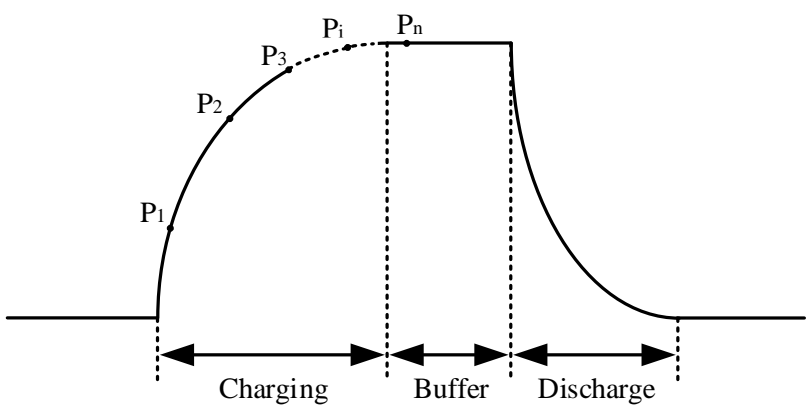

Figure 1. Principle of sampling in capacitance charge-discharge

Some problems need to be considered after the data of these sampling points are fitted. The first is the selection of the effective points. As can be seen from Figure 1, the time interval should be dealt with in the charging time range, but not all the sampling points are in this range. When the $\mathrm{Pi}$ point is on the left of the critical region, the effective point is $\mathrm{P}_{1} \sim \mathrm{P}_{\mathrm{i}}$; if the $\mathrm{P}_{\mathrm{i}}$ point is on the right side of the critical area, the effective point is selected as $\mathrm{P}_{1} \sim \mathrm{P}_{\mathrm{i}-1}$. Secondly, it is necessary to determine the time axis information of the sampling point. The time interval calculation can be obtained by the mutual elimination of the intermediate parameters. The actual measurement time is determined by the A/D sampling period and the number of sampling points. Thirdly, in the fitted curve model, the start time can be calculated by the voltage zero, which can be obtained without measurement. But the stop time is reflected by voltage at stop changing, so it can be extracted from the end of the sampled data. The obtained fitting curve model can be expressed as

$$
f=\left.F\right|_{\left(P_{1}, P_{2}, P_{3}, \ldots, P_{P}\right)}, \quad\left(0 \leq f \leq v_{n}\right)
$$

The final time interval can be expressed as

$$
\Delta T=\left.t\right|_{f\left(v_{n}\right)}-\left.t\right|_{f(0)}
$$




\section{EXPERIMENT AND ANALYSIS}

\subsection{Measurement and Results}

In order to verify the proposed method, the experiment is implemented on the basis of the circuit build in the Multisim software (Multisim Development Team, 2012) with actual parameters. In the circuit, the 8052 chip is used as the controller of the whole circuit. The ADC16 chip is a high-speed sampler, and the clock frequency is set to $10 \mathrm{MHz}$. The start-stop timing pulse signals are received by MUC respectively, and the corresponding operation is adopted to control the capacitance charge-discharge. As shown in Figure 2, the pin of $\operatorname{pin}_{1}$ and $\mathrm{pin}_{2}$ of MCU are connected with start-stop signal respectively. The $\operatorname{pin}_{15}$ and $\operatorname{pin}_{16}$ are connected with the switch (Q1 and Q2) of the capacitance charge-discharge respectively. The EN pin of ADC16 is connected with $\operatorname{pin}_{17}$, and the sampling data is transmitted through the connection of $\mathrm{P} 0 / \mathrm{P} 2$ and D0 D15. The capacitance charge-discharge is, firstly, set the $\operatorname{pin}_{15}=0, \operatorname{pin}_{16}=1$, $\operatorname{pin}_{17}=0$. When the start pulse is detected, $\operatorname{pin}_{15}=1, \operatorname{pin}_{16}=0$ and $\operatorname{pin}_{17}=1$ are set up, while P0/P2 read the sampled data. When the stop pulse is detected, $\operatorname{pin}_{15}=0, \operatorname{pin}_{16}=1$ and $\operatorname{pin}_{17}=0$ are set up, while P0/P2 stop reading the sampled data. Finally, the data collected in MCU are analysed and processed. In the experiment, the charging process at different time intervals is shown in Figure 3. The fixed start-stop interval is set to $4 \mu \mathrm{s}, 7$ $\mu \mathrm{s}, 12 \mu \mathrm{s}$ and $16 \mu \mathrm{s}$ through program in MCU. The frequency of the A/D sampler is $10 \mathrm{MHz}$. The value of $\mathrm{C}_{1}$ is $1 \mu \mathrm{F}$, and the charge impedance $R_{1}$ is $5 \Omega$. The corresponding time constant is $\tau=1 / \mathrm{R}_{1} \mathrm{C}_{1}=5 \mu \mathrm{s}$. Table 1 shows the effective points of the four capacitor charging curves obtained by sampling.

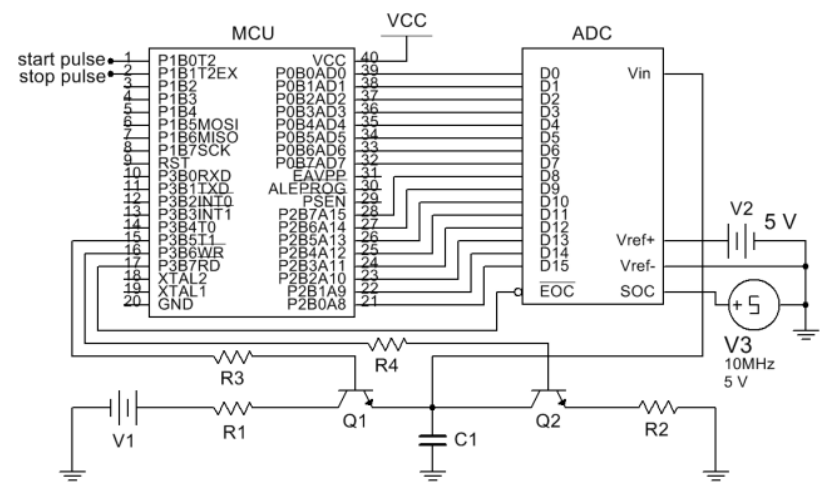

Figure 2. The main circuit model of time interval measurement based on capacitance charge-discharge

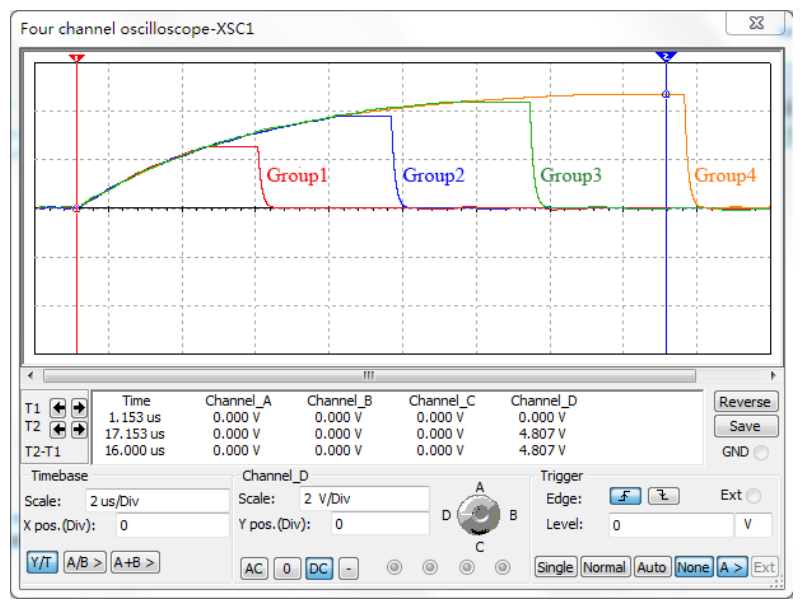

Figure 3. Capacitor charge-discharge at four time-intervals

\begin{tabular}{|l|lllllll|}
\hline & \multicolumn{7}{|c|}{ Voltage of the sampling point (V) } \\
\hline Group1 & 0.730 & 1.137 & 1.504 & 1.837 & 2.138 & 2.410 & 2.750 \\
\hline \multirow{2}{*}{ Group2 } & 0.730 & 1.137 & 1.504 & 1.837 & 2.138 & 2.410 & 2.657 \\
& 2.880 & 3.082 & 3.264 & 3.429 & 3.579 & 3.714 & 3.766 \\
\hline \multirow{4}{*}{ Group3 } & 0.730 & 1.137 & 1.504 & 1.837 & 2.138 & 2.410 & 2.551 \\
& 2.880 & 3.082 & 3.264 & 3.429 & 3.579 & 3.714 & 3.785 \\
& 3.947 & 4.047 & 4.138 & 4.220 & 4.294 & 4.361 & 4.572 \\
\hline \multirow{5}{*}{ Group4 } & 0.730 & 1.137 & 1.504 & 1.837 & 2.138 & 2.410 & 2.551 \\
& 2.880 & 3.082 & 3.264 & 3.429 & 3.579 & 3.714 & 3.785 \\
& 3.947 & 4.047 & 4.138 & 4.220 & 4.294 & 4.361 & 4.396 \\
& 4.477 & 4.527 & 4.572 & 4.613 & 4.649 & 4.683 & 4.807 \\
\hline
\end{tabular}

Table 1. Four Groups sampling point of capacitance voltage

\subsection{Data Analysis and Accuracy Evaluation}

The General model Exp2 is used to fit four sets of sampled point respectively in the processing of data. According to the fitting results in Figure 4, the goodness is as follows: the four SSE are less than $1 \%$, the four R-square are approximately 1 , and the four RMSE are all less than $1 \%$. Therefore, the fitting results can be regarded as a real relationship.
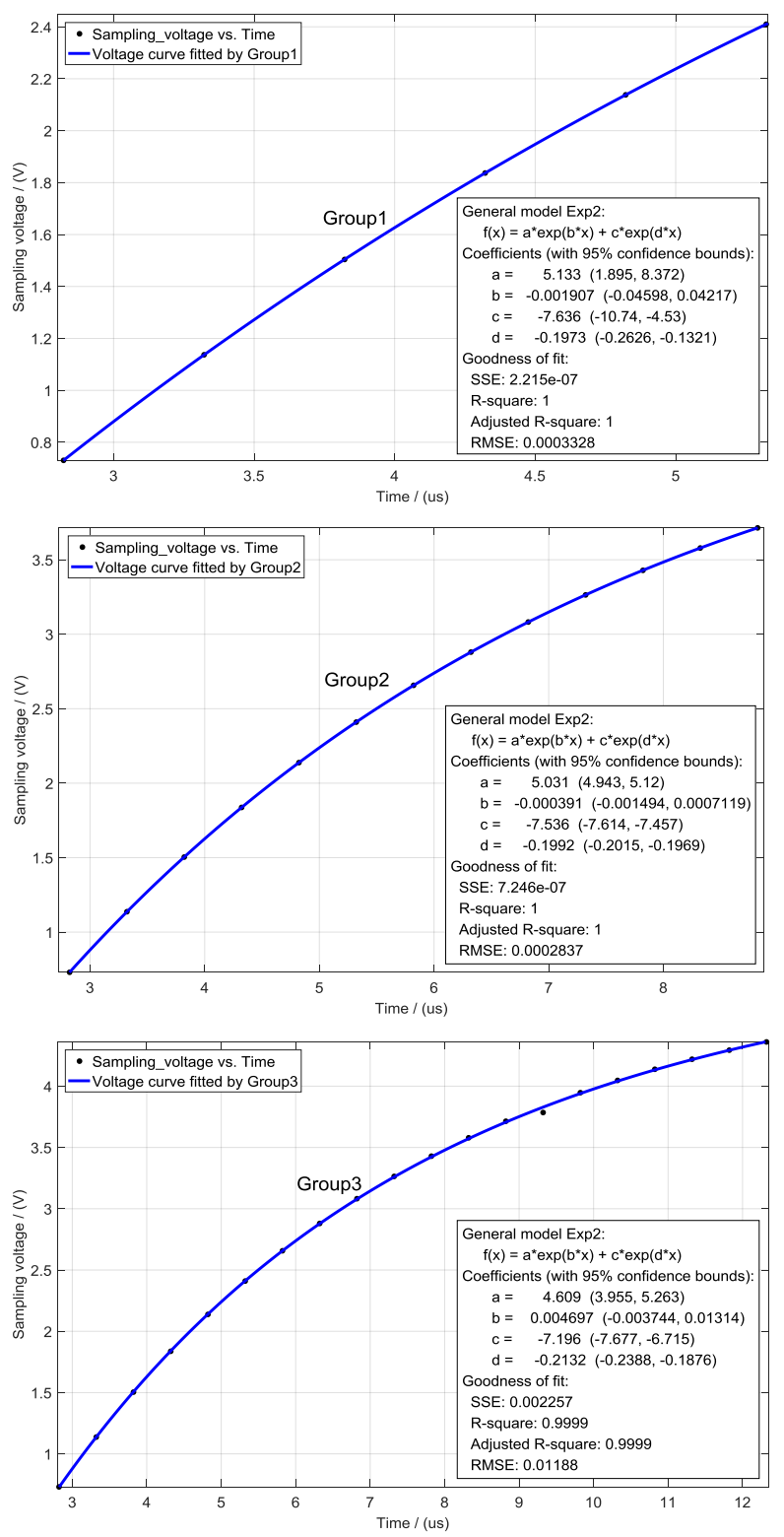


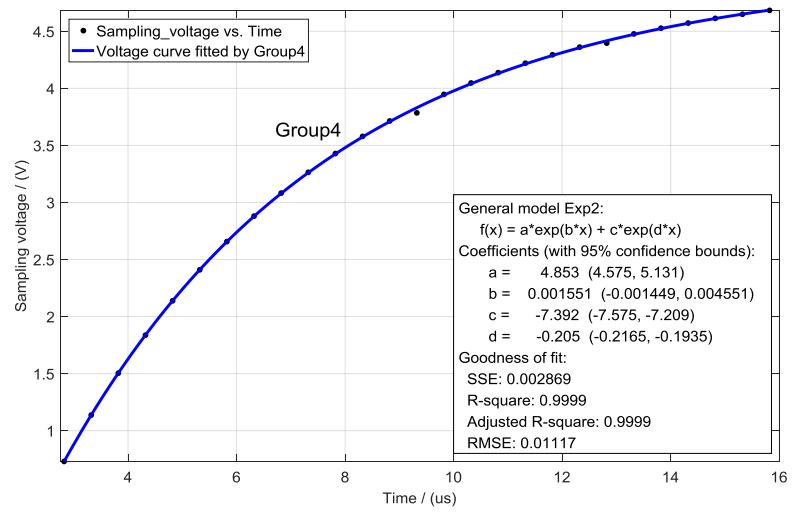

Figure 4. The fitting model of four group sampling points

Finally, the corresponding charging time and error (Table 2) can be calculated by the four models. Through the simulation experiment, the error is obtained, and it can be found that the more the number of sampling points, the smaller the error. In addition, the proposed method increases the accuracy by at least $20 \%$ compared with other methods (Table 3).

\begin{tabular}{|l|c|c|c|}
\hline Data & Design value $(\mu \mathrm{s})$ & Fitted values $(\mu \mathrm{s})$ & Error $(\mathrm{ps})$ \\
\hline Group1 & 4 & 4.0000764 & 76.4 \\
Group2 & 7 & 7.0000167 & 16.7 \\
Group3 & 12 & 12.0000035 & 3.5 \\
Group4 & 16 & 16.0000028 & 2.8 \\
\hline
\end{tabular}

Table 2 . The results and errors of the four sets of fitting models

\begin{tabular}{|l|c|}
\hline Methods & Accuracy (ps) \\
\hline Palojarvi (1999) & 3.5 \\
Nissinen (2009) & 30 \\
Klepacki (2014) & 7.5 \\
Our method & 2.8 \\
\hline
\end{tabular}

Table 3. Comparison of experimental accuracy with others

\section{CONCLUSION}

In this paper, a method based on capacitance charging and sampling fitting voltage curve is used to measure and improve the accuracy of time interval in pulse lidar. This method can avoid the nonlinear of electronic components and simplify the operation. Compared with others, the proposed method has higher accuracy and effect in remote sensing. In addition, highquality lidar remote sensing point cloud data with the accuracy error of $2.8 \mathrm{ps}$ is also available. The proposed method improves the accuracy over $20 \%$ when compared with other methods.

\section{ACKNOWLEDGEMENTS}

This paper is supported by the National Natural Science of China under Grant numbers 41431179, the National Key Research and Development Program of China under Grant numbers 2016YFB0502500, the State Oceanic Administration under Grant numbers 2014\#58, GuangXi Natural Science Foundation under Grant numbers 2015GXNSFDA139032, Guangxi Science \& Technology Development Program under the Contract numbers GuiKeHe 14123001-4, GuangXi Key Laboratory of Spatial Information and Geomatics Program under Grant numbers 151400701, 151400712, 163802512, Guangxi Young and Middle-aged Teachers Basic Ability Improvement Project under Grant numbers 2018KY0238,
Guangxi doctoral student innovation under Grant numbers YCBZ2018054.

\section{REFERENCES}

Acam messelectronic gmbh:Time-to-Digital-Converters, 2017. TDCs-Time-to-Digital Converters http://www.acam.de/products /time-to-digital-converters (9 December 2017)

Caulfield, H.J., 1986. A New Method for Measuring the Timeof-Flight in Fast Laser Range Finding. International Symposium/innsbruck. 654, pp. 19-25.

Gao, G.S. and Partridge, R., 1991. High speed digital TDC for D0 vertex reconstruction. IEEE Transactions on Nuclear Science. 38(2), pp. 286-289.

Kalisz, J., Szplet, R., Pelka, R. and Poniecki, A., 2002. Singlechip interpolating time counter with 200-ps resolution and 43-s range. IEEE Transactions on Instrumentation \& Measurement 46(4), pp. 851-856.

Klepacki, K., Szplet, R. and Pelka, R., 2014. A 7.5 ps singleshot precision integrated time counter with segmented delay line. Review of Scientific Instruments. 85(3), 034703.

Multisim Development Team, 2012. Interactive Image Technologies (Multisim) Software, Version 12.0.0. Analog/digital circuit simulation software http://www.ni.com (10 December 2017)

Nutt, R., 1968. Digital Time Intervalometer. Review of Scientific Instruments. 39(9), pp. 1342-1345.

Nissinen, J. and Kostamovaara, J., 2009. A $0.13 \mu \mathrm{m}$ CMOS laser radar receiver with leading edge detection and time domain error compensation. Instrumentation and Measurement Technology Conference, 2009. I2MTC '09. IEEE. pp. 900-903.

Palojarvi, P., Ruotsalainen, T. and Kostamovaara, J., 1999. A new approach to avoid walk error in pulsed laser rangefinding. IEEE International Symposium on Circuits and Systems. 1, pp. 258-261.

Zhou, G. and Xie, M., 2009. GIS-based Three-dimensional Morphologic Analysis of Assateague Island National Seashore from LIDAR Series Datasets. Journal of Coastal Research, 25(2), pp. 435-447.

Zhou, G., Zhou, X., Yang, J., Tao, Y., Nong, X. and Baysal, O., 2015. Flash Lidar Sensor using Fiber Coupled APDs. IEEE Sensor Journal, 15(9), pp. 4758-4768.

Zhou, G., and Zhou. X., 2014. Seamless Fusion of LiDAR and Aerial Imagery for Building Extraction. IEEE Transactions on Geoscience and Remote Sensing, 52(11), pp. 7393-7407.

Zhou, G., Song, C. and Schickler, W., 2004. Urban 3D GIS from LIDAR and aerial image data. Computers and Geosciences, 30(4), pp. 345-353.

Zieliński, M., Chaberski, D. and Grzelak, S., 2003. Timeinterval measuring modules with short deadtime. Komitet Metrologii I Aparatury Naukowej Pan. 10(3), pp. 241-251. 